\title{
"L'INFLUENCE DES LOGIQUES PÉNALES RÉTRIBUTIVES ET UTILITARISTES SUR LE NIVEAU D'ACCEPTATION DE LA RÉTENTION DE SÛRETÉ DANS LES CAS DE CRIMES PÉDOPHILES"
}

\author{
Manuel Tostain et Romain Lebreuilly \\ Presses universitaires de Liège | Les cahiers internationaux de psychologie sociale
}

2013/2 - Numéro 98

pages 237 à 257

ISSN 0777-0707

Article disponible en ligne à l'adresse:

http://www.cairn.info/revue-les-cahiers-internationaux-de-psychologie-sociale-2013-2-page-237.htm

Pour citer cet article :

Tostain Manuel et Lebreuilly Romain, « "L'influence des logiques pénales rétributives et utilitaristes sur le niveau d'acceptation de la rétention de sûreté dans les cas de crimes pédophiles" », Les cahiers internationaux de psychologie sociale, 2013/2 Numéro 98, p. 237-257.

Distribution électronique Cairn.info pour Presses universitaires de Liège.

(C) Presses universitaires de Liège. Tous droits réservés pour tous pays.

La reproduction ou représentation de cet article, notamment par photocopie, n'est autorisée que dans les limites des conditions générales d'utilisation du site ou, le cas échéant, des conditions générales de la licence souscrite par votre établissement. Toute autre reproduction ou représentation, en tout ou partie, sous quelque forme et de quelque manière que ce soit, est interdite sauf accord préalable et écrit de l'éditeur, en dehors des cas prévus par la législation en vigueur en France. II est précisé que son stockage dans une base de données est également interdit. 
G6

L'influence des logiques pénales rétributives et utilitaristes sur le niveau d'acceptation de la rétention de sûreté dans les cas de crimes pédophiles

Influence of retributive and utilitarian factors on the acceptance of civil commitment for pedophiles

Manuel TOSTAIN et Romain LEBREUILLY

Centre d'Etude et de Recherche sur les Risques et les

Vulnérabilités (CERReV, EA 3918), Université de Caen, France 
L'influence des logiques pénales rétributives et utilitaristes sur le niveau d'acceptation de la rétention de sûreté dans les cas de crimes pédophiles

Cette recherche avait pour objectif d'étudier l'influence des logiques pénales rétributives (faire payer le criminel) et utilitaristes (protéger la société) sur l'acceptation de la rétention de sûreté (placement en centre fermé, à l'issue de leurs peines, des criminels jugés dangereux). Auprès de 300 étudiants français, des informations relatives aux représentations des taux de récidive et des peines prononcées pour les crimes pédophiles ont été recueillies. 3 semaines plus tard, on leur a présenté le cas d'un pédophile en fin de peine. Deux facteurs étaient manipulés : (1) la longueur de sa peine initiale, (2) sa probabilité de récidive. Les sujets devaient ensuite indiquer s'il fallait le mettre en rétention. Les résultats montrent que la logique utilitariste est prédominante sur le niveau d'acceptation de la rétention de sûreté. En discussion, ces résultats sont mis en perspective avec les recherches de psychologie sociale de la justice et on aborde les questions sociétales que soulève cette disposition nouvelle.

\section{Influence of retributive and utilitarian factors on the acceptance of civil commitment for pedophiles}

A study was conducted to specify the respective influence of retributive (pay its debts) and utilitarian (protect the society) factors on civil commitment (placement in high security medical centers of dangerous individuals after their prison sentences). 300 french students read scenarios of a pedophile whose prison sentence had been completed. Two factors were manipulated: (a) his initial length of imprisonnement; ( b) his likelihood of recidivism. Participants had to indicate their acceptance of civil commitment for the pedophile. Information on their representations of recurrence rate and sentences for crimes pedophiles were previously collected. The experiment revealed that the utilitarian influence is predominant on the decision of civil commitement. In conclusion, researches on motivations of punishment were discussed and societal issues of this measure were raised.

Die Rolle der Rechtfertigung von Strafen als Vergeltung und als Schutz der Gesellschaft im Falle sexueller Vergehen: Die Frage der Sicherheitsverwahrung

Die Studie hatte das Ziel, die Rechtfertigung von Strafen als Vergeltung (retributive, für ein Vergehen bezahlen müssen) und als gesellschaftlichen Schutz (utilitariste) im Falle einer Sicherheitsverwahrung (Einweisung in Orte der Sicherheitsverwahrung nach Abschluss der Strafe für als gefährlich eingestufte Straftäter) zu untersuchen. Der Fall eines Sexualstraftäters wurde nach Abschluss der Strafvollzugszeit 372 französischen Studenten vorgestellt. Drei Faktoren auf dem mikrosozialen Level wurden manipuliert: (1) Die Länge der verhängten Strafvollzugszeit, (2) die Wahrscheinlichkeit eines Rückfalls und (3) seine Einstellung zur Aufnahme einer Therapie. Die Probanden sollten angeben, (1) ob ein Täter in eine Sicherheitsverwahrung eingewiesen werden sollte, (2) und ferner den Grad der Zustimmung im Hinblick auf einer allgemeinen Rechtfertigung des Strafmaßes (makrosozialer Level) nennen. Die Resultate zeigen, dass der Einfluss der Rechtfertigung einer Strafe als Vergeltung oder als Maßnahme des Schutzes der Gesellschaft für oder gegen eine Sicherheitsverwahrung je nach Mobilisierung eines mikro- oder makrosozialer Levels variiert. Abschließend lässt sich die Frage nach der sozialen Repräsentation sexuellen Vergehens und nach dem augenblicklichen Trend in der Sichtweise von Strafe stellen.

El rol de las justificaciones penales retributivas y utilitaristas en el caso de crímenes sexuales: la cuestión de la retención preventiva

Esta investigación tenía por objetivo estudiar las justificaciones penales retributivas (hacer pagar al criminal) y utilitaristas (proteger la sociedad) en el caso de la retención preventiva (colocación en un centro de detención, al final de las penas, de criminales calificados como peligrosos). Presentamos a 372 estudiantes franceses el caso de un criminal sexual terminando de cumplir su pena. Tres factores fueron manipulados (nivel micro social): (1) la duración de su pena inicial, (2) la probabilidad de 
recaída, (3) su actitud hacia un seguimiento psicoterapéutico. Los sujetos debían indicar: (1) si era necesario ponerlo en retención preventiva, (2) su grado de acuerdo con las justificaciones generales de la pena (nivel macro social). Los resultados mostraron que la influencia de las justificaciones penales retributivas y utilitaristas sobre la decisión de retención varían según el nivel micro o macro social movilizado. En conclusión, nos interrogamos sobre las representaciones sociales de la criminalidad sexual y las evoluciones penales actuales.

O papel das justificações penais retribuidoras e utilitaristas no caso de crimes sexuais: a questão da retenção de segurança

Esta pesquisa teve como objetivo estudar as justificações penais retribuidoras (fazer pagar o criminoso) e utilitaristas (proteger a sociedade) no caso de retenção de segurança (colocação no centro fechado, no final das suas sentenças, criminosos julgados perigosos). Apresentámos a 372 estudantes franceses o caso de um criminoso sexual no final da sua condenação. Três fatores foram manipulados (nível microssocial): (1) o tempo de sua sentença original, (2) a sua probabilidade de reincidência, (3) sua atitude perante um apoio psicoterapêutico. Os sujeitos foram convidados a indicar: (1) se se deve implementar a retenção, (2) o nível de acordo sobre justificações gerais de punição (nível macrossocial). Os resultados mostram que a influência de justificações penais retribuidoras e utilitaristas sobre a decisão de retenção varia de acordo com o nível micro ou macro mobilizado. Em conclusão, questionamos as representações sociais de crime sexual e atuais tendências penais.

Il ruolo delle giustificazioni penali retributive e utilitariste nel caso dei crimini sessulai : il problema della detenzione di sicurezza

Questa ricerca si poneva l'obiettivo di studiare le giustificazioni penali retributive (far pagare il criminale) e utilitariste (proteggere la società) nel caso della detenzione di sicurezza (internamento in un centro chiuso, alla fine della loro pena, dei criminali giudicati pericolosi). Abbiamo sottoposto a 372 studenti francesi il caso di un criminale colpevole di reati sessuali arrivato al termine della pena. Sono stati manipolati tre fattori (livello microsociale) : (1) la lunghezza della sua pena iniziale, (2) la probabilità di una sua recidiva, (3) il suo atteggiamento rispetto a una presa in carico psicoterapeutica. I partecipanti dovevano indicare : (1) se fosse necessario internarlo in una detenzione, (2) il loro grado di accordo sulle giustificazioni generali della pena (livello macrosociale). I risultati mostrano che l'influenza delle giustificazioni penali retributive e utilitariste sulla decisione a proposito della detenzione varia, a seconda del livello micro o macrosociale attivato. In conclusione, ci s'interroga sulle rappresentazioni sociali della criminalità di tipo sessuale e sugli sviluppi attuali delle pene. 
La correspondance pour cet article doit être adressée à Manuel Tostain, Université de Caen, UFR de Psychologie, Bureau SE 611, Campus 1, Esplanade de la paix, 14032 Caen, France ou par courrier à <manuel.tostain@unicaen.fr>. 


\section{Introduction}

Pourquoi punir les individus qui transgressent la loi ? Cette question, qui concerne toutes les sociétés, est traitée généralement par les philosophes et les juristes pénalistes (Carbasse, 2000 ; Fauconnet, 1920). En psychologie sociale, il existe néanmoins tout un courant d'étude sur la question de la justice et l'attribution de sanctions mais on remarque que les recherches s'intéressent davantage aux processus d'attribution de sanctions (le comment) qu'aux justifications de la sanction (le pourquoi) (Bordel, Guingouain, et Somat, 2006 ; Fincham et Jaspars, 1980 ; Oberlé et Gosling, 2004 ; Ross et Miller, 2002 ; Rainis, 2002 ; Shaver, 1985 ; Tostain, 2007 ; Weiner, 1995). Dans ce contexte, l'objectif de la recherche était d'étudier les effets des facteurs associés aux différentes justifications pénales dans le cas des crimes pédophiles, crimes qui soulèvent dans l'opinion publique une émotion très grande. L'étude de la façon dont les individus envisagent le traitement de ces crimes est importante car s'y révèle l'état d'esprit du corps social à un moment donné et s'y illustre les tensions, les débats sur les évolutions pénales actuelles qui vont dans un sens toujours plus répressif (Przygodzki-Lionet, 2012 ; Salas, 2010). Dans cette recherche, nous souhaitions étudier l'importance des justifications pénales dites rétributives (faire payer le criminel pour le mal qu'il a commis) par rapport aux justifications pénales dites utilitaristes (isoler le criminel pour protéger la société). Pour cela, nous avons choisi de nous référer à une disposition pénale qui est étroitement associée à la pédophilie, la rétention de sûreté. Cette mesure consiste à placer dans des centres médico-judiciaires fermés, à l'issue de leurs peines, les individus jugés encore dangereux, c'est-à-dire pour lesquels on considère qu'il y a une forte probabilité de récidive. Plusieurs raisons nous ont conduit à choisir cette disposition pour étudier les justifications pénales des sujets. D'abord, cette disposition est exemplaire des tensions entre logiques rétributives et logiques utilitaristes. D'un côté, elle se situe dans une logique rétributive car elle revient, en introduisant une privation de liberté supplémentaire, à faire payer plus fortement le criminel. D'un autre côté, elle s'inscrit dans une logique utilitariste car elle a pour objectif de protéger la société contre la survenue d'éventuels nouveaux crimes en maintenant emprisonnés les criminels jugés dangereux. Cette disposition est également importante à étudier car elle donne lieu actuellement à des débats très vifs tant chez les juristes que dans I'opinion publique. Cette disposition a en effet été récemment introduite dans le code pénal français ${ }^{1}$. Ses opposants y voient une peine supplémentaire injuste pour des criminels qui sont sur le point de finir de payer leur dette à la société, tandis que ceux qui y sont favorables mettent en avant la nécessité de disposer de mesures permettant d'éviter de nouvelles victimes. Cette mesure pose donc la question de l'équilibre entre différents principes politiques et démocratiques: le respect des droits et libertés individuelles des condamnés d'un côté, la responsabilité de l'état de garantir la sécurité de ses concitoyens d'un autre côté. En définitive, nous avons eu l'objectif de mieux cerner les logiques pénales qui influencent l'acceptation ou le refus de cette mesure aux enjeux sociétaux importants. Nous avons par ailleurs choisi de nous centrer sur les 
crimes pédophiles car ces crimes provoquent des attitudes contrastées dans I'opinion publique quant aux mesures de justice à mettre en œuvre et c'est principalement en réaction à ce type de crimes que cette mesure de rétention de sûreté a été décidée (Mucchielli, 2011, Salas, 2010).

\subsection{Les justifications pénales}

La philosophie pénale identifie habituellement deux grandes catégories de justifications de la peine à même d'influencer le jugement et l'attribution de sanction (Vidmar et Miller, 1980 ; Villey, 2003). La première catégorie renvoie à la perspective dite rétributive (" just deserts theory » selon la terminologie anglo-saxonne) (Hegel, 1821/1982 ; Kant, 1796/1971). Selon cette perspective, I'homme a une volonté libre et doit en conséquence répondre de ses actes. $\mathrm{S}^{\prime} \mathrm{il}$ commet un crime, il doit souffrir à hauteur du mal qu'il a commis, ce qui se traduit par un principe de proportionnalité entre la gravité du crime commis et la sévérité de la sanction (Carlsmith, 2006 ; McFatter, 1978). Dans ce sens, les différents codes pénaux prévoient des peines d'emprisonnement d'autant plus grandes que les crimes sont jugés graves par la société. Par cette peine, le criminel paye sa dette à la société. La seconde catégorie de justifications renvoie à la perspective dite utilitariste ( deterrence theory » selon la terminologie anglo-saxonne) (Beccaria, 1764/1991 ; Bentham, 1789/1802). Ici la sanction est une réaction du corps social à un crime qui constitue une violation et une menace pour l'ordre social. Elle a pour but de protéger la société en empêchant l'auteur du crime de récidiver ou de dissuader d'autres individus de commettre les mêmes infractions. La sanction se justifie par ses conséquences potentiellement bénéfiques pour la société : elle sera d'autant plus légitime qu'elle permet de réduire la probabilité de survenue de nouveaux crimes, de limiter la récidive (Carlsmith, Darley, et Robinson, 2002 ; De Keijser, Van der Leeden, et Jackson, 2002 ; Oswald, Hupfeld, Klug, et Gabriel, 2002).

Parmi les spécialistes du droit pénal (Van de Kerchove, 2005 ; Viney, 2000), la prédominance de l'une par rapport à l'autre philosophie pénale dans la détermination de la sanction fait l'objet d'un débat récurrent. Ce débat est également présent dans les études de psychologie sociale de la justice (Robinson et Darley, 1997). De nombreuses études montrent que c'est la justification rétributive qui est la plus importante pour les individus ordinaires, en mettant en évidence que ce sont les facteurs rétributifs - importance du mal commis, souffrance de la victime, caractère moralement répréhensible du crime - qui influencent le plus la sanction (Carlsmith et al., 2002 ; Darley, Carlsmith, et Robinson, 2000 ; Feather, 2002). Pour autant, certaines études soulignent, à l'inverse, que les facteurs associés à la perspective utilitariste, tels la fréquence du crime, la dangerosité future du criminel, sont plus déterminants (Graham, Weiner, et Zucker, 1997 ; Rucker, Polifroni, Tetlock, et Scott, 2004 ; Weiner, Graham, et Reyna, 1997). Dans ce cadre, une recherche de Carlsmith, Monahan et Evans (2007) apporte une contribution importante en faveur de la prédominance de la perspective rétributive dans les jugements ordinaires. Dans cette recherche, Carlsmith et al. se sont intéressés à la rétention de sûreté (en anglais " civil commitment »). Cette disposition, ainsi qu'on I'a dit plus 
haut, consiste à maintenir en détention le criminel à l'issue de sa peine si, suite à des expertises, on considère qu'il présente des risques élevés de récidive. Cette disposition est clairement utilitariste, sa mise en œuvre ayant pour but d'empêcher que des individus jugés encore dangereux pour la société ne soient remis en liberté et commettent de nouveau des crimes (Harris, 2005). Carlsmith et al., dans leur recherche, ont présenté aux sujets le cas d'un pédophile en fin de peine et les sujets devaient décider ou non d'une mesure de rétention de sûreté à son encontre. Les auteurs manipulaient conjointement les facteurs rétributif et utilitariste. Selon les conditions, la peine initiale était soit « insuffisante » (3 ans) car trop faible selon le principe rétributif de proportionnalité entre gravité du crime et importance de la sanction, soit «suffisante » (25 ans) selon les normes de jugements américains. Pour étudier l'effet de la logique utilitariste, ils faisaient varier la probabilité estimée de récidive du criminel à l'issue de sa peine : soit elle était nulle $(\mathrm{O} \%)$, faible (4\%) ou très élevée $(70 \%)$. Carlsmith et al. montrent que l'acceptation de cette disposition est surtout déterminée par le facteur rétributif. Ainsi, les sujets recommandent d'autant plus cette mesure que la peine initiale est « insuffisante ». Pour Carlsmith et al. (2007), les sujets cherchent ainsi à rétablir le principe rétributif de proportionnalité de la peine, la rétention de sûreté - en prolongeant la détention - constituant un moyen de compenser l'insuffisance de la peine initiale. Par ailleurs, ils constatent que les sujets ne prennent en compte la logique utilitariste que lorsque la logique rétributive a été respectée : c'est seulement quand la peine initiale est "suffisante » qu'ils tiennent compte de la probabilité de récidive. Dans ce cas, mais seulement dans ce cas, ils recommandent d'autant plus la rétention que la probabilité de récidive est élevée. Sinon, en cas de peine "insuffisante ", ils recommandent fortement cette mesure quel que soit le niveau de récidive du criminel. Dès lors, pour ces auteurs, cette recherche indique clairement que la logique rétributive est prédominante par rapport à la logique utilitariste puisque la première est davantage présente que la seconde et ce, même pour des dispositions pénales a priori utilitaristes comme la rétention de sûreté.

Pour autant, on peut s'interroger sur le degré de généralisation de cette recherche qui a été faite dans un contexte socio-juridique particulier, à savoir le contexte américain. Il convient de rappeler que les pays occidentaux relèvent de traditions juridiques pour parties différentes. Ainsi, les Etats-Unis (et la plupart des pays anglo-saxons) s'inscrivent dans ce que les juristes appellent la « common law » (on parle aussi de système accusatoire), tandis qu'un certain nombre de pays européens notamment " latins », telle la France, s'inscrivent dans le cadre de la " civil law » (ou système inquisitoire). Or, les juristes s'accordent à dire que les pays de " common law », et spécialement les Etats-Unis, sont davantage rétributifs que les pays de " civil law » comme la France (Garapon, Gros, et Pech, 2001 ; Pradel, 2002). Ainsi, si on se réfère à l'intensité de la peine, dimension centrale de la logique rétributive, celle-ci est bien plus forte aux Etats-Unis qu'en France. Dans ce sens, si on compare, aussi bien le guide américain de détermination des peines (Hinojosa, 2007 ; Rossi, Berk, et Campbell, 1997) et le code pénal français d'une part (Wackenheim, 2008), que les statistiques officielles de condamnations par 
type de crimes des deux pays d'autre part (United States Sentencing Commission, 2011 ; Ministère Français de la Justice, 2010), on note que les peines sont bien moins sévères en France qu'aux Etats-Unis². En définitive, on est en droit de se demander si la focalisation sur la dimension rétributive est bien aussi importante dans des pays aux traditions juridiques différentes. L'objectif de la recherche était donc de vérifier cette prédominance de la logique rétributive dans un pays européen tel que la France, au système de « civil law ».

Nous voulions également préciser les facteurs rétributifs et utilitaristes impliqués dans l'acceptation de cette mesure. En particulier, on peut se demander si les représentations qu'ont les sujets des taux de récidive et des peines ne jouent pas un rôle. Ainsi, dans une optique utilitariste de protection de la société, la nécessité de cette mesure de rétention peut paraître d'autant plus légitime que les sujets considèrent que les probabilités de récidive des pédophiles sont importantes, I'enjeu d'éviter de nouvelles victimes devenant plus impératif. Toujours dans cette logique utilitariste, une autre question concerne la représentation que les sujets ont du niveau à partir duquel la probabilité de récidive constitue un risque majeur à ne pas négliger. Pour certains individus, vu le caractère intolérable de ce type de crime, il ne faut prendre aucun risque, un risque de $1 \%$ étant encore un risque de trop. Pour d'autres, on doit malgré tout accepter, tolérer un certain risque car une fois que le criminel a payé sa dette, il a le droit de recouvrer la liberté. De plus, exiger en pareil cas un risque quasi nul voire un risque zéro impliquerait de prendre le risque complémentaire de laisser en détention nombre de criminels qui, une fois qu'ils auraient payé leurs dettes, n'auraient pas récidivé, ce qui serait injuste. Ces différentes façons d'envisager le risque " tolérable » ne sont peut-être pas sans influence sur l'acceptation de la rétention de sûreté. Par exemple, on peut penser que les sujets davantage fixés sur un risque zéro ${ }^{3}$, considérant comme problématique la récidive dès des seuils de probabilité faibles, soient plus favorables à cette mesure de rétention afin de limiter ce risque. C'est ce que nous avons cherché à vérifier.

La représentation que les sujets se font de ce qu'est une peine juste et des pratiques des tribunaux mérite aussi d'être abordée. Cette question touche à la perception de la justice et aux débats qui lui sont associés, certains accusant cette institution d'être trop laxiste, d'autres dénonçant au contraire sa nature de plus en plus répressive (Mucchielli, 2011). Dans le cadre de la logique rétributive de proportionnalité des peines en fonction des crimes, les individus souhaitent qu'il y ait une correspondance entre ce qui leur semble être juste en terme de peines et les pratiques effectives de la justice en matière de condamnation. Si les individus pensent qu'il y a des écarts entre leurs conceptions de la peine juste et les peines prononcées par les tribunaux, on peut penser que cela puisse influencer leur acceptation de la rétention de sûreté. Par exemple, si les peines effectives sont, d'après eux, en deçà de la peine juste, ils seront peut-être plus favorables à la rétention de sûreté, cette mesure pouvant être une manière de compenser, en prolongeant la détention, une sanction des tribunaux jugée insuffisante. Nous avons donc également cherché à vérifier cette hypothèse. 
En définitive, dans le contexte d'un pays européen de " civil law », la France, I'objectif de la recherche était d'étudier l'impact des facteurs rétributifs et utilitaristes sur l'acceptation de la rétention de sûreté dans le cas de crimes pédophile. Pour ce faire, nous avons procédé à un questionnement des sujets sur leurs représentations de la récidive et de la peine (partie 1 de l'étude) et nous avons mis en œuvre une procédure expérimentale inspirée de la recherche de Carlsmith et al. (2007) mais adaptée à notre pays (partie 2 de l'étude)Hypothèses

Hypothèse 1 : en accord avec la logique rétributive, on s'attendait à ce que la mesure de rétention de sûreté soit davantage acceptée quand la peine serait « insuffisante » (peine faible) que lorsque la peine serait «suffisante » (peine forte). En cas de peine " insuffisante ", la rétention de sûreté en prolongeant la détention, permettrait de rétablir la logique rétributive de proportionnalité entre gravité du crime et intensité de la peine, (effet principal de la longueur de la peine).

Hypothèse 2 : en accord avec la logique utilitariste de la rétention de sûreté qui a pour objectif de protéger la société des individus dangereux, on s'attendait à ce que cette mesure soit d'autant plus acceptée que la probabilité de récidive serait élevée (effet principal de la probabilité de récidive).

Hypothèse 3 : en référence aux résultats de Carlsmith et al. (2007), on postulait une prédominance de la logique rétributive sur la logique utilitariste. En condition peine " insuffisante », on s'attendait à ce que la logique rétributive domine : les sujets recommanderaient fortement la rétention de sûreté afin de compenser l'insuffisance de la peine initiale et ce, sans tenir compte de la probabilité de récidive. On s'attendait à ce que la logique utilitariste ne soit mobilisée uniquement quand la dimension rétributive aurait été respectée, c'est-à-dire en cas de peine «esuffisante ». Dans cette situation, l'acceptation de la rétention de sûreté serait d'autant plus forte que la probabilité de récidive serait élevée (effet d'interaction longueur de la peine x probabilité de récidive). On s'attendait néanmoins à un effet $d^{\prime}$ 'interaction plus modéré que dans l'expérience de Carlsmith et al. (2007), la France présentant une tradition juridique moins fortement rétributive que les Etats-Unis.

Hypothèse 4 : elle concernait l'impact des représentations des probabilités de récidive sur la rétention de sûreté, cette hypothèse s'inscrivant dans la logique utilitariste. On s'attendait à ce que les sujets soient d'autant plus favorables à la rétention de sûreté que : 4a leurs estimations des probabilités réelles de récidive seraient élevées ; $4 b$ : le taux à partir duquel ils jugeraient grave le risque de récidive serait faible.

Hypothèses 5 : elle setréférait au principe de proportionnalité de la logique rétributive. Elle concernait l'effet, sur l'acceptation de la rétention de sûreté, des représentations des peines jugéesejustes au regard des estimations des peines réelles prononcées par les tribunaux. On s'attendait à l'effet suivant : plus les estimations des durées des peines moyennes prononcées par les tribunaux français seraient inférieures aux durées des peines estiméesejustes par les sujets et plus ceux-ci seraient favorables à la rétention de sûreté : la rétention de sûreté - en tant que peine supplémentaire -permettant de réduire l'écart entre peines réelles estimées et peines jugées justes. 


\section{Méthode}

\subsection{Sujets}

300 étudiants de premier cycle en sciences sociales ont participé à l'étude (211 femmes et 89 hommes d'une université située dans une ville de taille moyenne de l'ouest de la France, 200.000 habitants). L'expérience s'est déroulée en deux parties séparées de 3 semaines. La passation était collective, en groupe d'une vingtaine de personnes. La première partie était présentée comme une étude sur les représentations de la délinquance et de la justice, la seconde partie comme une étude sur les jugements en matière pénale.

Première partie : questionnaire initial : les taux de récidive et les durées des peines

Les sujets ont été invités à remplir un questionnaire d'une trentaine de questions sur les différents types de délinquance et le fonctionnement de la justice. Parmi ces questions, quatre concernaient directement notre recherche : il était demandé aux sujets d'indiquer pour le viol d`un mineur : (1) ce qu'ils considéraient êtr: un risque de récidive constituant un problème grave pour la société, (2) d'estimer la probabilité réelle de récidive pour ce type de crime (en indiquant à chaque fois un pourcentage allant de 0 à 100). Ensuite, ils devaient indiquer (en années de prison), pour le viol dıun mineur : (3) la peine qui leur paraissaitejuste pour un tel crime, (4) la peine moyenne prononcée d'après eux par les juridictions françaises. À la fin du questionnaire, ils remplissaient une fiche signalétique dans laquelle ils indiquaient leur sexe et leur opinion politique (sur une échelle en 7 points allant de 1 : tout à fait à gauche à 7 : tout à fait à droite).

Seconde partie (3 semaines plus tard) : protocole expérimental

Les sujets lisaient une note préliminaire reprenant les dispositions légales françaises de la rétention de sûreté : "Cette mesure consiste à placer, à l'issue de leur peine de prison, les individus condamnés pour crimes dans des centres médico-judiciaires fermés s'ils sont considérés encore dangereux par un panel d'experts psychiatres. Dans ces centres, ils bénéficient d'une prise en charge psychothérapeutique. Cette mesure, d'une durée initiale d'un an, peut-être renouvelée indéfiniment, tant que l'individu représente une menace pour la société en terme de dangerosité ». Puis, sur la même feuille, ils prenaient connaissance d'un fait divers décrivant un criminel sexuel âgé de 40 ans condamné pour le viol de deux fillettes âgées de 9 ans. Il était indiqué qu'il était juste à la fin de sa peine de prison ${ }^{4}$.

\subsection{La manipulation de la longueur de la peine initiale}

Dans la condition peine «faible» («einsuffisante»), le criminel avait été condamné à 5 ans de prison, dans la condition peine " forte » («esuffisante») à 20 ans.

\subsection{La manipulation de la probabilité de récidive}

Les sujets apprenaient que peu avant sa prochaine libération un panel de trois experts psychiatriques spécialisés en criminalité sexuelle avait estimé sa proba- 
bilité de récidive s'il était amené à être libéré. Selon les conditions, cette probabilité était de $0 \%{ }^{5}$ (probabilité nulle), de $5 \%$ (probabilité faible), ou de $40 \%$ (probabilité élevée).

Les valeurs des modalités de la variable longueur de la peine initiale et de la variable probabilité de récidive ont été choisies suite à un pré-test sur 40 étudiants (20 femmes et 20 hommes) en sciences sociales à qui il a été demandé d'indiquer pour ce type de crime : 1) une peine prononcée par les tribunaux qui était d'après eux injuste car trop faible (en années) ; 2) une peine prononcée par les tribunaux qui leur paraissait juste (en années) ; 3) une probabilité de récidive qu'ils jugeaient faible ; 4) une probabilité de récidive qu'ils jugeaient élevée. Pour déterminer les valeurs de nos modalités (peine faible, forte, probabilités faible et élevée), nous avons pris les médianes obtenues à chacune des quatre questions du pré-test.

Les sujets indiquaient ensuite sur une échelle en 7 points de type Likert (de $1=$ tout à fait opposé à $7=$ tout à fait favorable) s'ils étaient ou non favorable à la mise en place d'une mesure de rétention de sûreté dans le cas du criminel présenté.

\section{Résultats}

\subsection{Première partie de la recherche : représentations par les sujets des probabili- tés de récidives et des attributions de peines}

Cette analyse avait pour but d'étudier les représentations par les sujets des probabilités de récidive des pédophiles et des peines justes et attribuées par les tribunaux français. La probabilité estimée de récidive est de 51,3\% $(E . T .=26,74)$. Ces estimations varient beaucoup, les valeurs s'échelonnant entre $8 \%$ et $82 \%$. On peut dire que les sujets surestiment fortement la récidive pour ce type de crime puisque les chiffre réels sont d'environ 10\% (Kensey et Tournier, 2004 ; Zochetto, 2007). Les femmes font une estimation un peu plus élevée de cette récidive que les hommes $F(1,298)=13,9 ; p<.002$. Mfemmes $=56,5 ; E . T .=27,23$. Mhommes $=45,20:$ $E . T .=24,92$. La probabilité à partir de laquelle le risque de récidive constitue un problème grave est de $18,52 \%(E . T .=15,55)$. II n'y a pas d'effet du sexe $F(1,298)$ $=0,59 ; p<.44, n s$.

Concernant les peines, la peine moyenne que les sujets estiment être donnée par les tribunaux français en cas de viol sur mineur est de 10,29 années (E.T. = $6,15)$. Cette estimation est assez proche de la réalité, la peine moyenne en la matière étant d'après les statistiques officielles de 9 ans (Ministère Français de la Justice, 2010). L'estimation par les femmes des peines réelles attribuées est un peu inférieure à celle des hommes $F(1,298)=5,24 ; p<.02$. Mfemmes $=9,54$; $E . T .=6,70$. Mhommes $=11,16 ; E . T .=5,33)$. Le nombre d'années de prison pour que la peine soitejuste est en moyenne de 21,92 années $(E . T .=15,69)$ mais cela varie selon les sujets, cette estimation s'échelonnant entre 5 et 60 années de prison, environ $15 \%$ ajoutant sur le questionnaire la peine de mort comme peine juste. Cette peine considérée comme juste est en moyenne plus élevée et varie davantage pour les femmes que pour les hommes $F(1,298)=9,43 ; p<.002$. Mfemmes $=24,46 ; E . T .=19,08$. Mhommes $=18,98$. E.T. $=9,52$. On note donc 
que la peine considérée comme juste $(21,92)$ est en moyenne plus élevée eplus du double) que l'estimation de la peine prononcée par les tribunaux $(10,29)$.

\subsection{Deuxième partie de la recherche : effet des manipulations expérimentales : longueur de la peine, probabilité de récidive}

Nous avons procédé à une analyse de variance : 2 (longueur de la peine : faible, forte) X 2 (probabilité de récidive : nulle, faible, élevée) X 2 (sexe des sujets), avec comme variable dépendante la note d'acceptation de la rétention de sûreté (qui allait de 1 : tout à fait opposé à 7 : tout à fait favorable).

Il n'y a pas d'effet principal de la longueur de la peine sur l'acceptation de la rétention $F(1,288)=1,19 ; p<.27$; ns (pour plus de détails, voir le tableau 1 ). Contrairement à ce qui était attendu selon l'hypothèse 1 qui se référait à la logique rétributive, l'acceptation de la mesure de rétention n'est pas plus forte quand la peine initiale est faible que lorsqu'elle est forte. II y a par contre un effet principal de la manipulation de la probabilité de récidive $F(2,288)=62,37, p<.0001$. Cet effet est important, I'Eta-deux partiel ayant une valeur de 0,3). Plus précisément, et conformément à l'hypothèse 2 , I'acceptation de la rétention de sûreté est d'autant plus importante que la probabilité de récidive est élevée (Mproba nulle $=4,13$; $E . T .=2,08$. Mproba faible $=5,45 ; E . T .=1,60$. Mproba élevée $=6,66 ; E . T .=0,79$ ). Le test post-hoc de Scheffé indique que ces trois moyennes sont significativement différentes les unes des autres (Mproba nulle - Mproba faible $=: p<.0001$. Mproba faible - Mproba élevée $=p<.0001$ ). Contrairement à ce qui était attendu selon I'hypothèse 3 , il n'y a pas d'effet d'interaction longueur de la peine $X$ probabilité de récidive $F(2,288)=0,44 ; p<.64$. $n s$. On ne note pas d'effet principal $(F(1,288)$ $=0,15 \mathrm{p}<.0,70 . n s)$ ni d'effets d'interaction du sexe des sujets.

\subsection{L'analyse de régression multiple}

Afin de préciser l'incidence des logiques rétributive et utilitariste sur l'acceptation de la rétention de sûreté nous avons procédé à une analyse de régression multiple, en prenant en compte les représentations des probabilités de récidives et des peines par les sujets ( $1{ }^{\text {ère }}$ partie de la recherche) et les variables manipulées du dispositif expérimental ( $2^{\text {nde }}$ partie de la recherche). Dans la $1^{\text {ère }}$ partie de la recherche, pour préciser

Tableau 1 : Notes moyennes d'acceptation de la mise en rétention du criminel selon la longueur de la peine initiale et la probabilité de récidive (de 1 : tout à fait opposé ; à 7 : tout à fait favorable) (entre parenthèses : écarts-types)

\begin{tabular}{lccc}
\hline & Peine de 5 ans & Peine de 20 ans & \\
\hline Probabilité de récidive $0 \%$ & $4,30(1,98)$ & $3,96(2,18)$ & $4,13(2,08)$ \\
Probabilité de récidive $5 \%$ & $5,60(1,45)$ & $5,30(1,74)$ & $5,45(1,60)$ \\
Probabilité de récidive 40\% & $6,64(0,80)$ & $6,68(0,79)$ & $6,66(0,79)$ \\
\hline & $5,51(1,76)$ & $5,31(2,00)$ & $5,41(1,88)$ \\
\hline
\end{tabular}


I'influence de la logique utilitariste, nous avons pris comme prédicteurs la probabilité réelle de récidive estimée par les sujets et le taux de probabilité à partir duquel la récidive est jugée grave. On s'attendait à ce que les sujets soient d'autant plus favorables à la rétention de sûreté qu'ils estimeraient la probabilité réelle de récidive importante, cette criminalité étant perçue dans cette configuration comme plus dangereuse (hypothèse 4a). De même, on s'attendait à ce que les sujets soient d'autant plus favorables à la rétention de sûreté que les niveaux de probabilités à partir desquels ils considèreraient la récidive comme un problème grave seraient bas (hypothèse $4 b$ ) : un niveau d'autant plus bas pouvant en effet laisser penser que les sujets sont d'autant plus sensibles aux risques de récidive. Pour préciser l'influence de la logique rétributive, à partir des données de la 1 ère partie de la recherche, nous avons construit une nouvelle variable correspondant à la différence entre l'estimation de la peine que les sujets considèrent juste pour ce type de crime et leurs estimations des peines prononcées par les tribunaux : plus l'écart sera important et plus cela signifiera que les sujets tendent à trouver injustes, au regard de leurs positions personnelles, les peines prononcées par les tribunaux. Plus précisément, si l'écart est négatif, cela signifiera que les sujets trouvent trop fortes les peines prononcées, si l'écart est positif, cela signifiera que les sujets trouvent les peines prononcées insuffisantes. Dès lors, si on suit une logique rétributive, on s'attendait à ce que les sujets soient d'autant plus favorables à la rétention de sûreté que l'écar, entre l'estimation de la peine juste et l'estimation de la peine prononcée par les tribunau, serait grand et irait dans un sens positif (cette mesure pouvant être vue alors comme une façon de compenser cette insuffisance des peines prononcées, en prolongeant la détention du criminel). En définitive, nous avons intégré cinqtprédicteurs : les deux manipulations de la partie 2 expérimentale : (1) la longueur de la peine et (2) la probabilité de récidive, les trois informations issues de la partie 1 de la recherche : (3) les probabilités estimées des récidives réelles, (4) le niveau des probabilités à partir desquelles la récidive est jugée grave, (5) notre nouvelle variable correspondant à la différence entre peines jugées justes et peines prononcées par les tribunaue. La variable dépendante était la note d'acceptation de la rétention de sûreté (qui allait de 1 : tout à fait opposé à 7 : tout à fait favorable).

La part de variance expliquée par l'analyse de régression multiple sur l'acceptation de la rétention de sûreté est de $42 \%\left(R^{2}\right) F(7,292)=30,54 ; p<.0001$. deux prédicteurs en rapport avec la logique utilitariste ont un effet sur l'acceptation de la rétention de sûreté : il y a ainsi un effet de la manipulation expérimentale de la probabilité de récidive $(t(292)=12,26 ; p<.0001 ; \beta=+55)$ et un effet de l'estimation par les sujets de la probabilité de récidive réelle $(t(292)=6,06 ; p<.0001 ; \beta=+0,30)$ : plus la probabilité de récidive manipulée est élevée (hypothèse 2 )t plus également la probabilité estimée de récidive réelle est forte (hypothèse 4a), et plus l'acceptation de la rétention de sûreté est élevéé. Par contre, il n'y a pas d'effets : (1) de la longueur de la peine $(t(292)=$ $1,13 ; p<.25$. ns; $\beta=+0,05$. Hypothèse 1$)$, (2) du niveau à partir duquel la probabilité de récidive est jugée grave $(t(292)=0,53 ; p<.81$. $n s ; \beta=+0,01$. Hypothèse $4 b)$, (3) ni de la différence entre l'estimation de la peine juste et l'estimation des peines prononcées par les tribunaux $\left(t(292)=1,15 ; p<.28 \text {. ns ; } \beta=+0 ; 06^{6} \text {. Hypothèse } 5 b\right)^{7}$. 


\section{Discussion}

Dans nos sociétés contemporaines, la question de l'insécurité et de son traitement fait l'objet de débats importants (Lagrange, 2003). Dans ce cadre, la criminalité pédophile tient une place particulière. Elle soulève une émotion particulièrement forte dans I'opinion publique (Garapon et Salas, 2006) et en réaction des dispositions pénales de plus en plus sévères, telle la rétention de sûreté que nous avons étudiée ici, sont apparues dans le paysage pénal (Salas, 2010). L'objectif de notre recherche était de préciser l'influence des logiques pénales rétributives et utilitaristes sur l'acceptation de cette mesure dans le contexte de la France, pays de « civil law».

Aàpropos de l'importance ds ces deus logiques pénales, nos résultats montrent que la logique utilitariste est prédominante par rapport à la logique rétributive. Ainsi, on note un effet important d'une dimension centrale de la logique utilitariste, le niveau de probabilité de récidive. D'une part, on remarque un impact de la représentation de la récidive pédophile (partie 1 de la recherche) sur le niveau d'acceptation de la rétention : plus les sujets considèrent que cette criminalité présente des taux de récidives élevés et plus ils recommandent cette mesure. D'autre part, plus le niveau de probabilité de récidive manipulé est élevé (partie 2 de la recherche) et plus les sujets recommandent également la rétention de sûreté. Toutefois, le niveau à partir duquel la probabilité de récidive est jugée grave n'a pas d'effet sur l'acceptation de la rétention de sûreté. Cette mesure est peut-être moins en rapport avec la logique utilitariste qui se centre principalement sur le niveau de récidive (de faible à élevé). Cette notion de gravité est peut-être aussi pluseambiguë, moins courante pour les sujets que la notion de probabilité élevée. On remarque que les facteurs en rapport avec la logique rétributive ne présentent pas d'effes. D'abord, si on s'intéresse aux représentations des sujets ( 1 ère partie de la recherche), on ne note pas d'effet, en terme d'acceptation de la rétention, de l'écart entre leurs estimations des durées des peines qu'ils considèrent comme justes et leurs estimations des durées des peines réelles prononcées par les tribunaux. Une logique rétributive aurait incité à penser que plus les sujets font des estimations des durées justes plus longues que ce qu'ils pensent être les durées réelles prononcées, et plus ils verraient cet écart injuste. Ce écari les amènerait à être plus favorable à la rétention, celle-ci pouvant être un moyen de le compenset en rallongeant la détention. Tel n'est donc pas le cas. Au niveau de la manipulation expérimentale ( $2^{\text {nde }}$ partie de la recherche) on ne remarque également pas d'effets associés au principe de proportionnalité de la justice rétributive : I'acceptation de la rétention ne varie pas selon le caractère «esuffisant» (peine forte) ou "einsuffisant » de la peine (peine faible). Ces résultats sont différents de ceux de Carlsmith et al. (2007) qui constataient au contraire une prédominance de la logique rétributive sur la logique utilitariste. Ainsi, leurs sujets tenaient compte de la probabilité de récidive uniquement quand la logique rétributive était " satisfaite », c'est-à-dire quand la peine était "esuffisante ». Sinon, en cas de peine "einsuffisante ", la rétention de sûreté était fortement acceptée, quel que soit le niveau de récidive. Un tel écart entre leur recherche et la nôtre est à interpréter avec prudence car les deux recherches n'ont pas les mêmes spécificités : 
ce n'est pas la même population, cinq années se sont écoulées entre les deux recherches, et les valeurs des modalités expérimentales ne sont pas toutes similaires. Signalons toutefois un point qui pourrait expliquer pour partie cette plus grande focalisation dans la recherche de Carlsmith et al. (2007) sur le facteur rétributif. Dans leur recherche, dans la condition peine « insuffisante », il était indiqué que le criminel était dans une prison très "libre », à la sécurité minimale et tout confort ${ }^{8}$. Cette information décrivant des conditions de détention très positives peut être problématique car cela revient à cumules dans une même condition expérimentale deux dimensions pouvant mobiliser le facteur rétributif : (1) la longueur insuffisante de la peine initiale et (2) le fait que la prison, en raison de son grand confort, ne fait pas suffisamment payer au criminel sa dette à la société. Cette double information, alors qu'il n'y en a qu'une dans leur recherche pour le facteur utilitariste (le niveau de récidive) a pu orienter leurs résultats en incitant les sujets à se focaliser davantage sur le facteur rétributif que sur le facteur utilitariste. C'est pour éviter ce possible déséquilibre entre les deux facteure dans notre manipulation expérimentale, que nous avons d'ailleurs décidé de supprimer cette information sur le confort de la prison dans notre étude

Si nous cherchons maintenant à mettre en perspective notre recherche avec ce qui est généralement constaté dans les études sur les logiques pénales à l'œuvre dans les jugements ordinaires (De Keijser et al., 2002 ; McFatter, 1978 ; PrzygodzkiLionet, 2012), contrairement à ce qui est souvent relevé dans les travaux de psychologie sociale de la justice (Carlsmith et al., 2002; Oswald et al., 2002), dans notre recherche c'est la logique utilitariste qui prédomine sur la logique rétributive. Pour expliquer cette différence, il faut d'abord préciser que la plupart de ces travaux sont issus de pays anglo-saxons et majoritairement des Etats-Unis. Or, comme nous l'avons indiqué plus haut, ces pays s'inscrivent dans une tradition juridique différente de la nôtre. Ce sont des pays de "common law » tandis que la France, et un certain nombre de pays européens, sont des pays de " civil law ». De plus, comme les juristes s'accordent à le dire (Garapon et Papadopoulos, 2003), les Etats-Unis sont un pays plus axé sur la rétribution que la France. Cette tendance plus rétributive aux Etats-Unis se manifeste par : (1) le principe du cumul des peines qui peut conduire à des emprisonnements très longs ${ }^{9}$ (alors qu'en France c'est le principe de non-cumul ou de confusion des peines qui prévaut en cas de peines multiples, la durée totale d'emprisonnement correspond à la durée de la peine la plus longue) et : (2) à crimes équivalents, les peines y sont plus bien plus sévères. Cette différence peut aussi s'expliquer par la spécificité du crime choisi dans notre recherche, la pédophilie, où la logique utilitariste est très présente. Ce crime, qui suscite une émotion très forte, pose en effet de façon aigue le problème des risques de récidives, l'évitement de nouvelles victimes étant un enjeu sociétal important (Archer, 1998). Cette différence peut aussi s'expliquer par la nature mixte de la rétention de sûreté : à la fois sanction et mesure de soin. Or, les travaux sur les justifications pénales s'intéressent généralement à des mesures qui sont uniquement des sanctions. Enfin, il n'est pas impossible que le poids de la logique rétributive ait été sous-estimé dans les résultats de notre recherche. Nous avons interprété l'effet 
constaté des probabilités de récidive sur l'acceptation de la rétention de sûreté comme le signe de la forte présence de la logique utilitariste. II est vra, que, par définition, plus ce risque est élevé et plus le criminel présente un danger pour la société qui doit s'en protéger, ce qui est le propre de la logique utilitariste. Pour autant, une forte probabilité de récidive peut être aussi envisagée dans une optique rétributive. L'auteur du crime, s'il paraît prêt à recommencer, à récidiver, peut-être perçu comme quelqu'un qui n'a toujours pas compris, assumé et regretté I'horreur de son acte. Ce criminel peut-être vu dans ce cas comme manifestant une insensibilité coupable à l'égard d'autrui ${ }^{10}$, comme quelqu'un d'inhumain qui mérite, dans une optique rétributive, un châtiment plus grand ${ }^{11}$. Pour conclure sur cet écart entre la tendance des travaux à souligner la prédominance de la logique rétributive sur la logique utilitariste et notre recherche où c'est l'inverse qui se produit, on peut dire que cette prédominance de l'utilitarisme que nous constatons s'explique également par le fait que notre sanction pénale, la rétention de sûreté, par rapport à d'autres sanctions pénales, a cette caractéristique d'avoir pour fondement de protéger la société en prévenant la récidive. Lors de la présentation aux sujets de cette mesure, les conditions d'application légale qui étaient rappelées correspondaient à cet objectif utilitariste.

Notre recherche comporte cependant des limites. En particulier, ellexa été faitt sur une population étudiante. On doit donc être prudent quant à la généralisation de nos résultats. Pour autant, ces résultats ne sauraient être considérés comme nécessairement non représentatifs. Il y a d'abord chez nos sujets étudiants une tendance similaire à celle que l'on trouve dans les échantillons représentatifs de la population française. Cette même tendance consiste à surestimer fortement les probabilités réelles de récidive en matière de pédophilie (Mucchielli, 2011). Ensuite, certaines recherches montrent que les variables sociales (niveau d'éducation, de revenu, type de profession, etc.) ne sont pas toujours strictement liées aux opinions sur les justifications pénales de la peine (Hamilton et Rytina, 1980 ; Rossi et al., 1997), et dans la recherche déjà citée de Carlsmith et al. (2007), les auteurs ne trouvèrent pas de différences significatives entre les résultats sur un échantillon d'étudiants en sciences sociales et un échantillon de citoyens éligibles au statut de jurés qui était représentatif de la population générale.

La rétention de sûreté est une disposition qui se développe dans les pays européens et qui est nouvelle pour la France (introduite en 2008). A I'issue de notre recherche, il nous semble important de rappeler qu'elle pose questions tant sur le plan juridique que sociétae (De Beaurepaire, Bénézech, et Kottler, 2004 ; Janus, 2006 ; Winick et La Fond, 2003), et qu'elle fait l'objet d'un débat important entre opposants et tenants de cette mesure. Pour les opposants à la rétention de sûreté, tels I'ancien garde des Sceaux Robert Badinter ${ }^{12}$ (2007), cette mesure est inacceptable car elle enfreint les principes du droit moderne. Le premier de ces principes est que I'individu ne doit être puni que pour le crime qu'il a commis. Avec cette mesure, les individus sont punis (la rétention constituant une peine privative de liberté) pour un crime futur et hypothétique, pour leur supposée dangerosité, le lien normalement exigé entre le crime commis et sa conséquence, la peine, disparaissant. Il y a 
là, qui plus est, pour les opposants, un risque sérieux d'arbitraire et d'erreurs car le savoir psychiatrique, n'étant pas un savoir scientifique au sens prédictif du terme, il ne permet pas d'estimer avec assurance la dangerosité future d'un individu. On risque donc de garder en prison des criminels qui n'auraient pas récidivé. Cette mesure contrevient également au second principe du droit pénal qui énonce que nul ne saurait être puni deux fois pour le même acte. Pour les opposants, cette mesure modifie l'esprit de la justice. On passe d'un système centré sur la responsabilité individuelle et sur ce qui a été commis à un système de protection de la société où des individus peuvent être maintenus quasi indéfiniment en détention (cf. l'optique de l'école criminologique italienne. Ferri, 1893/2004). Elle constitue, en définitive, pour les opposants une violation des droits individuels qui ne serait pas sans faire penser à la justice de ces états totalitaires décrits dans la littérature (e.g. "Le procès » de Fran. Kafka. 1925) ou au cinéma (e.g. " Minority report » de Steve. Spielberg. 2002). Pour les tenants de la rétention de sûreté, cette disposition est au contraire légitime car la société a le droit et le devoir de protéger la société des individus dangereux, d'éviter de nouvelles victimes : c'est un impératif social et moral (Lecerf, 2008). Ils rétorquent à ceux qui contestent la rétention de sûreté qu'il ne s'agit pas d'une peine mais d'une mesure de protection. Ils précisent que dans le contexte français, I'individu n'est pas maintenu en prison mais envoyé dans un centre médico-judiciaire fermé dans lequel il bénéficie d'une prise en charge médico-psychologique systématique afin de le soigner et de l'aider à modifier son comportement. Elle a donc aussi une dimension réhabilitative, de soutien à la réinsertion future de l'individu dans la société. Ils ajoutent que cette restriction de liberté n'est que temporaire car cette mesure est révisable tous les ans et offre toutes les garanties d'un jugement équitable (décision d'un juge après avis psychiatriques et débat contradictoire entre l'accusation et la défense). Au regard des arguments des uns et des autres, on peut dire que cette disposition pose la question de l'équilibre à trouver entre liberté, droits des individus condamnés et droit à la sécurité de la société. Remarquons qu'en France elle fait l'objet d'un fort assentiment, $80 \%$ de la population y étant favorable (Institut Français d'Opinion Publique, 2008). Cet assentiment important, malgré les problèmes qu'elle soulève, est comme le suggère notre recherche peut-être pour partie associe à une surestimation des taux de récidives réees des criminels sexuels par la population. Rappelons que dans notre échantillon, nous avons constaté que le taux de récidive réel était estimé à $50 \%$ alors qu'il " $\mathrm{n}^{\prime}$ est » en réalité que d'environ $10 \%$. Or, nous avons mis en évidence que plus les sujets estimaient ce taux de récidive important et plus ils étaient favorables à la rétention de sûreté. Vu les enjeux de cette disposition, et les débats auxquels elle donne lieu, il nous paraît important d'étudier pour la suite plus précisément comment les individus dans les pays européens envisagent cette disposition (comme une mesure de soin, comme une peine supplémentaire ?). Ajoutons pour conclure, que cette mesure de rétention de sûreté sera certainement de nouveau au centre de l'actualité française, la nouvelle majorité de gauche élue en 2012 ayant le projet de supprimer cette disposition mise en place par la précédente majorité de droite. 


\section{Notes}

1. Loi n²008-174 du 25 Février 2008.

2. Même si la France connaît ces dernières années un durcissement de sa politique pénale (Salas, 2010).

3. Cette focalisation sur le risque zéro relevant peut-être de variables psychosociales (croyances en un monde juste, sentiment de menace, Montada et Lerner, 2010).

4. Nous n'avons pas repris certaines informations des vignettes de Carlsmith et al. (2007). Par exemple, nous avons supprimé dans la condition peine « insuffisante » l'information qui indiquait que le prisonnier était dans une prison à la sécurité minimale et tout confort. Dans le contexte français où l'état des prisons est très dégradé, ces informations auraient été peu crédibles (rapport de l'Observatoire International des Prisons, 2011). De plus, comme nous l'évoquerons en discussion, cette information soulève quelques questions.

5. Pour rendre plausible cette probabilité nulle, on précisait dans cette condition que le condamné prenait un médicament supprimant toutes les pulsions sexuelles (castration chimique).

6. Le $\beta$ est également non significatif si on prend, à la place de notre nouvelle variable " différence peine juste-peine prononcée », la variable initiale " estimation de la peine juste par les sujets »: $\mathrm{t}(292)=0,74 ; p<.45$. ns. $\beta=+0,05$.

7. Une analyse complémentaire montre un léger effet de l'orientation politique $(\mathrm{t}(292)=$ $2,16 ; \mathrm{p}<.03 ; \beta=+0,12)$ : plus les sujets se positionnent à droite et plus ils ont tendance à être favorables à la rétention de sûreté.

8. «A confortable minimum-security prison with full access to sports, movies, libraries, and visitors ».

9. Par exemple, dans l'affaire «Madoff » du nom de ce financier qui avait escroqué ses clients, le principal protagoniste a été condamné à 150 ans de prison.

10. On remercie l'un des experts d'avoir attiré notre attention sur ce point.

11. Tel le personnage de Meursault dans le livre «L'étranger» d'Albert Camus (1942), qui, pour l'homicide qu'il a commis, est condamné à la peine de mort car les jurés le trouvent inhumain et insensible (la cour est révoltée qu'il n'ait pas eu d'émotion lors du décès de sa mère).

12. Qui a joué un grand rôle en France dans I'abolition de la peine de mort en 1981. 


\section{Bibliographie}

- Archer, E. (1998). Agressions sexuelles : victimes et auteurs. Paris : Editions L'Harmattan.

- Badinter, R. (2007). La prison après la peine. Le Monde daté du 28 novembre, 20-21.

- Beccaria, C. (1991). Traité des délits et des peines. Paris : Flammarion. (première publication 1764).

- Bentham, J. (1802). Traité de législation civile et pénale. Paris : Bossange et Besson. (première publication 1789).

- Bordel, S., Guingouain, G., \& Somat, A. (2006). Objective and subjective responsibility in a judicial context. Swiss Journal of Psychology, 65(4), 227-235.

- Camus, A. (1942). L'étranger. Paris : Gallimard.

- Carbasse, J.M. (2000). Histoire du droit pénal et de la justice criminelle. Paris : Presses Universitaires de France.

- Carlsmith, K.M. (2006). The roles of retribution and utility in determining punishment. Journal of Experimental Social Psychology, 42, 437-451.

- Carlsmith, K.M, Darley, J.M., \& Robinson, P.H. (2002). Why do we punish ? Deterrence and just deserts as motives for punishment. Journal of Personality and Social Psychology, 83, 284-299.

- Carlsmith, K.M., Monahan, J., \& Evans, A. (2007). The function of punishment in the "civil" commitment of sexually violent predators. Behavioral Sciences \& the Law, 25, 437-448.

- Darley, J.M., Carlsmith, K.M, \& Robinson, P.H. (2000). Incapacitation and just deserts as motives for punishment. Law and Human Behavior, 24, 659-683.

- De Beaurepaire, C., Bénézech, M., \& Kottler, C. (2004). Les dangerosités : de la criminologie à la psychopathologie, entre justice et psychiatrie. Paris : John Libbey Eurotext.

- De Keijser, J.W., Van der Leeden, R., \& Jackson, J.L. (2002). From moral theory to penal attitudes and back: A theoretically integrated modeling approach. Behavioral Sciences and the Law, 20, 317-335.

- Fauconnet, P. (1920). La responsabilité. Paris : Felix Alcan.

- Feather, N.T. (2002). Deservingness, entitlement, and reactions to outcomes. In M. Ross \& D.T. Miller (Eds.), The justice motive in everyday life (pp. 334-349). Cambridge: Cambridge University Press.

- Ferri, E. (2004). La sociologie criminelle. Paris : Dalloz((première publication 1893).

- Fincham, F.D., \& Jaspars, J.M. (1980). Attribution of responsibility: From man the scientist to man as lawyer. In L. Berkowitz (Ed.), Advances in Experimental Social Psychology (vol.13, pp. 81-138). New-York: Academic Press.

- Garapon, A., Gros, F., \& Pech, T. (2001). Et ce sera justice : punir en démocratie. Paris : Editions Odile Jacob.

- Garapon, A., \& Papadopoulos, I. (2003). Juger en France et en Amérique. Paris : Editions Odile Jacob.

- Garapon, A., \& Salas, D. (2006). Les nouvelles sorcières de Salem : leçons d'Outreau. Paris : Le Seuil.

- Graham, S., Weiner, B., \& Zucker, G.S. (1997). An attributional analysis of punishment goals and public reactions to O.J. Simpson. Personality and Social Psychology Bulletin, 23, 331-346. 
- Hamilton, V.L., \& Rytina, S. (1980). Social consensus on norms of justice: Should the punishment fit the crime? American Journal of Sociology, 85, 1117-1144.

- Harris, A.J. (2005). Civil commitment of sexual predators: A study in policy implementation. London: Scholarly Publishing.

- Hegel, F. (1982). Principes de la philosophie du droit. Paris : Vrin. (première publication 1821).

- Hinajosa, R.H. (2007). United States Sentencing Guidelines Manual. Washington DC: United States Houses of Representatives.

- Janus, E.S. (2006). Failure to protect: America's sexual predator law and the rise of preventive state. Cornell: Cornell University Press.

- Kafka, F. (1987). Le procès. Paris : Gallimard. (première publication 1925).

- Kant, E. (1971). Doctrine du droit. Paris : Vrin. (première publication 1796).

- Kensey, A, \& Tournier, P.V. (2004). La récidive des sortants de prison. Cahiers de démographie pénitentiaire, $n^{\circ} 15$.

- Lagrange, H. (2003). Demandes de sécurité. Paris : Le Seuil.

- Lecerf, J.R. (2008). Rapport sénatorial n¹74 sur le projet de loi relatif à la rétention de sûreté et à la déclaration d'irresponsabilité pénale pour cause de trouble mental. Paris : La Documentation Française.

- McFatter, R.M. (1978). Sentencing strategies and justice: Effects of punishment philosophy on sentencing decisions. Journal of Personality and Social Psychology, 36, 1490-1500.

- Ministère Français de la Justice (2010). Les condamnations en 2005. Paris : La Documentation Française.

- Montada, L., \& Lerner, M.J. (2010). Response to victimizations and belief in a just world. New-York : Springer-Verlarg.

- Mucchielli, L. (2007). Violences et insécurité : fantasmes et réalités dans le débat français. Paris : La Découverte.

- Mucchielli, L. (2011). L'invention de la violence, des peurs, des chiffres, des faits. Paris : Fayard.

- Oberlé, D., \& Gosling, P. (2004). Intention, responsabilité, sanction : I'intention est-elle un préalable ou une justification de la sanction ? In J.L. Beauvois, R.V. Joulé \& J.M. Monteil (Eds.), Perspectives Cognitives et Conduites Sociales ( vol. 9, pp. 271-192). Rennes : Presses Universitaires de Rennes.

- Observatoire International des Prisons (2011). Les conditions de détention en France. Paris : Editions La Découverte.

- Oswald, M.E., Hupfeld, J., Klug, \& Gabriel, U. (2002). Lay-perspectives on criminal deviance, goals of punishment, and punitivity. Social Justice Research, 15, 85-98.

- Pradel, J. (2002). Droit pénal comparé. Paris : Dalloz.

- Przygodzki-Lionet, N. (2012). Psychologie et justice : de l'enquête au jugement. Paris : Dunod.

- Rainis, N. (2002). Les contributions de la psychologie judiciaire et de l'expertise psychojuridique à l'administration de la justice pénale. In A. Leblanc, M. Doraï, N. Roussiau \& C. Bonardi (Eds.), Psychologie sociale appliquée : Education, Justice, Politique (pp. 91-116). Paris: In Press Editions. 
- Robinson, P.H., \& Darley, J.M. (1997). The utility of desert. Northwestern University Law Review, 91, 453-499.

- Ross, M., \& Miller, D.T. (2002). The justice motive in everyday life. Cambridge: Cambridge University Press.

- Rossi, P.H., Berk, R.A., \& Campbell, A. (1997). Just punishments: Guidelines sentences and normative consensus, Journal of Quantitative Criminology, 13, 267-290.

- Rucker, D.D., Polifroni, M., Tetlock, P.E., \& Scott, A.L. (2004). On the assignment of punishment: The impact of general-societal threat and the moderating role of severity. Personality and Social Psychology Bulletin, 30, 673-684.

- Salas, D. (2010). La volonté de punir : essai sur le populisme pénal. Paris : Hachette.

- Shaver, K.G. (1985). The attribution of blame: Causality, responsibility, and blameworthiness. New-York: Springer-Verlag.

- Tostain, M. (2007). L'influence des motivations à punir sur les jugements de responsabilité et I'attribution de sanction aux auteurs d'infractions pénales. Cahiers Internationaux de Psychologie Sociale, 75-76, 35-49.

- United States Sentencing Commission (2011). Statistical Information Packet. Washington DC: United States Houses of Representatives.

- Van de Kerchove, M. (2005). Les fonctions de la sanction pénale. Informations Sociales, $127,22-31$.

- Vidmar, N, \& Miller, D. (1980). Social psychological processes underlying attitudes toward legal punishment. Law and Society Review, 14, 565-602.

- Villey, M. (2003). La formation de la pensée juridique moderne. Paris : Presses Universitaires de France.

- Viney, G. (2000). La responsabilité et ses transformations (responsabilités civile et pénale), In Y. Michaud (Ed.), L'université de tous les savoirs, vol.2 : qu'est-ce que l'humain ? (pp. 144156). Paris : Editions Odile Jacob.

- Wackenheim, V. (2008). Le code pénal. Paris : Editions Prat.

- Weiner, B. (1995). Judgments of responsibility: A foundation for a theory of social conduct. New-York: Guilford Press.

- Weiner, B., Graham, S., \& Reyna, C. (1997). An attributional examination of retributive versus utilitarian philosophies of punishment. Social Justice Research, 10, 431-451.

- Winick, B.J., \& La Fond, J.Q. (2003). Protecting society from sexually dangerous offenders: Law, justice, and therapy. Washington DC: American Psychological Association.

- Zochetto, F. (2007). Rapport sénatorial n³58 sur le projet de loi renforçant la lutte contre la récidive des majeurs et des mineurs. Paris : La Documentation Française. 